\title{
Population Regulation and Character Displacement in a Seasonal Environment
}

\author{
Emma E. Goldberg, ${ }^{1, \star}$ Russell Lande, ${ }^{2}$ and Trevor D. Price ${ }^{3}$ \\ 1. Department of Biological Sciences, University of Illinois, Chicago, Illinois 60607; 2. Division of Biology, Imperial College London, \\ Silwood Park Campus, Ascot, Berkshire SL5 7PY, United Kingdom; 3. Department of Ecology and Evolution, University of Chicago, \\ Chicago, Illinois 60637
}

Submitted November 6, 2011; Accepted February 21, 2012; Electronically published April 25, 2012

\begin{abstract}
Aвstract: Competition has negative effects on population size and also drives ecological character displacement, that is, evolutionary divergence to utilize different portions of the resource spectrum. Many species undergo an annual cycle composed of a lean season of intense competition for resources and a breeding season. We use a quantitative genetic model to study the effects of differential reproductive output in the summer or breeding season on character displacement in the winter or nonbreeding season. The model is developed with reference to the avian family of Old World leaf warblers (Phylloscopidae), which breed in the temperate regions of Eurasia and winter in tropical and subtropical regions. Empirical evidence implicates strong winter density-dependent regulation driven by food shortage, but paradoxically, the relative abundance of each species appears to be determined by conditions in the summer. We show how population regulation in the two seasons becomes linked, with higher reproductive output by one species in the summer resulting in its evolution to occupy a larger portion of niche space in the winter. We find short-term ecological processes and longer-term evolutionary processes to have comparable effects on a species population size. This modeling approach can also be applied to other differential effects of productivity across seasons.
\end{abstract}

Keywords: character displacement, competition, Phylloscopus warblers, population regulation, quantitative genetics, seasonal environment.

\section{Introduction}

Interspecific competition has both ecological and evolutionary consequences for population size. First, resource appropriation by one species reduces individual fecundity or survival of the other, with the result that population size is lower than it would be in the absence of the competitor (Schoener 1983). Second, competition results in evolutionary divergence between species, a process termed "ecological character displacement" (Brown and Wilson 1956; Grant 1972; Slatkin 1980), which will usually result

* Corresponding author; e-mail: eeg@uic.edu.

Am. Nat. 2012. Vol. 179, pp. 693-705. (C) 2012 by The University of Chicago. 0003-0147/2012/17906-53435\$15.00. All rights reserved.

DOI: $10.1086 / 665649$ in additional changes in population size (Slatkin 1980; Taper and Case 1985). Ecological character displacement is considered to be widespread in nature and an important determinant of morphological and ecological differences between sympatric species (Schluter 2000, chap. 6; Dayan and Simberloff 2005; Pfennig and Pfennig 2009). The standard theory, as developed for two species, predicts that character displacement should occur when the range of resources consumed by each species is sufficiently constrained relative to the width of available resources (Slatkin 1980; Taper and Case 1985; Doebeli 1996). For equivalent competitors, the population size of each species is lower at equilibrium than it would be in the absence of the competitor, not only because of resource depression but because each species has evolved to a position in resource space where the resources are relatively low (see fig. 1). Here, the range of resources utilized by one species is the so-called realized niche (Chase and Leibold 2003), but the fundamental niche (i.e., the range of resources that would be consumed if the competitor were absent) is also affected by phenotypic displacement. Models of character displacement are thus one way to model evolution of the niche (Roughgarden 1972; Taper and Case 1985).

Most species that follow an annual cycle experience different resource distributions and resource requirements at different seasons. Intuitively, if the quantity of resources available to a species differs between seasons, population size should be under greater density-dependent regulation in one season than the other. Here we show how this intuition breaks down when evolution is included. Specifically, we model two competing species and demonstrate that a species with access to a greater abundance of resources in one season evolves to exploit a greater abundance of resources in the other season. The net result is that both abundant and rare species can be under strong density-dependent regulation throughout the year.

We first describe an empirical system, that of the Old World leaf warblers (Phylloscopidae), where cross-seasonal effects appear to have affected population regulation. 

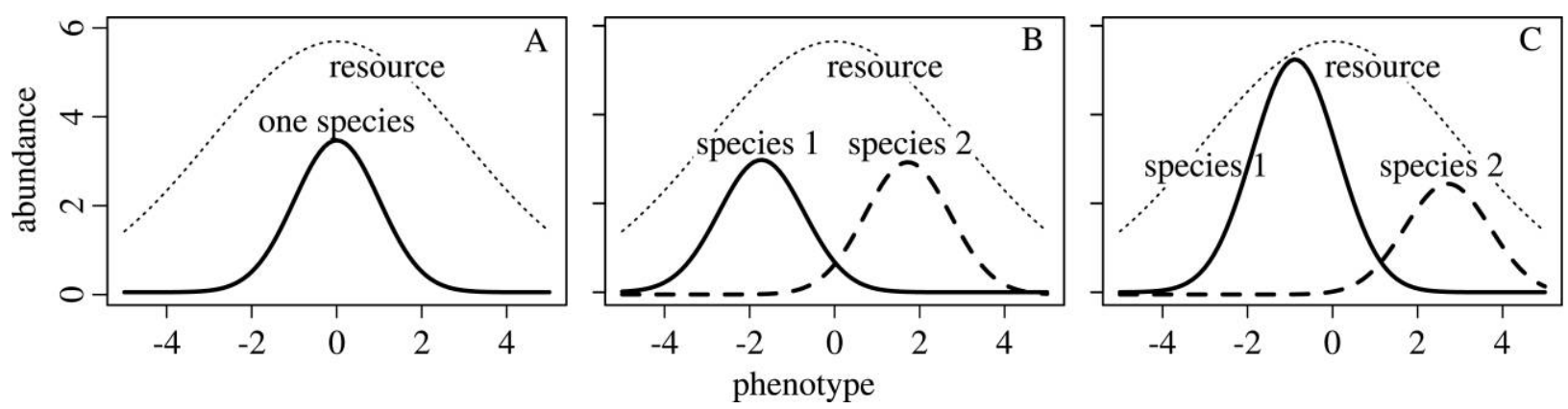

Figure 1: Equilibrium distributions of competing species (solid and dashed lines) under a unimodal resource distribution (thin dotted line). A, A single species. $B$, A pair of competing species with identical properties except for their mean phenotypes. $C$, A pair of species that are equivalent in all respects except that the carrying capacity in the summer for species 1 is 2.5 times higher than the carrying capacity for species 2. Parameter values for $C$ are $K_{1}^{(s)}=50, K_{2}^{(s)}=20, r^{(s)}=2.0, h^{2}=0.5, r^{(w)}=-0.5, Q_{0}=10, \sigma_{Q}^{2}=9, \sigma_{z}^{2}=\sigma_{a}^{2}=1, a_{i j}=1$.

Twenty-one species of Phylloscopidae have populations breeding somewhere in the Himalayas in the summer (Rasmussen and Anderton 2005). Among these 21, entire populations of several western species overwinter in India, and these have been especially well studied. In the winter, behavioral observations (Price 1981; Katti and Price 1996), along with correlations of food and warbler densities across both space and time $(r \sim 1.0)$, indicate strong densitydependent population regulation (Katti and Price 1996; Gross and Price 2000; Price and Gross 2005). This implies that the relative abundances of different species (i.e., differences in the total numbers of individuals per species) should be largely determined by winter conditions. It is clear, however, that relative abundances depend on the summer breeding season, not the winter nonbreeding season. Those species that breed at high elevations in the Himalayas track climate and habitat into Siberia, with the result that they have much larger population sizes than species found at low elevations, which are confined to the Himalayan region (Price et al. 1997). The presence of strong winter limitation but relative abundances determined by conditions in the summer season seems paradoxical, because if each species were independently regulated in its own ecological niche in winter, then relative abundances should be largely determined by that season.

A possible resolution of this paradox is that species are ecologically identical in the winter, so that their abundances depend only on differential output from the summer season (similar to neutral ecological models). However, this is patently not the case; species are very different in their ecology. A particularly important axis of niche differentiation among coexisting species in winter is foraging height (Gross and Price 2000; Price and Gross 2005), which is associated with morphological differences. In north India in the winter, among the two common species, Phylloscopus humei has a relatively long tarsus and forages in the tree crowns, whereas Phylloscopus chloronotus has a shorter tarsus and forages in the bushes. The presence of more open vegetation in the treetops favors more hopping and less flying in search of food, and this likely explains the association of foraging height with tarsus length (Price and Gross 2005).

Given strong evidence for food limitation, we consider that the ecological differences between this pair of species are maintained by interspecific competition for food. It is possible, however, that character displacement was not involved in the production of the differences, and instead winter communities have been built up by sorting of ecologically differentiated species. Furthermore, other factors, most notably different suites of competitors, the light environment, and probably many other factors including predators and parasites, increase adaptation of each species to its preferred foraging heights (Gross and Price 2000). However, both P. humei and P. chloronotus do regularly forage at each other's favored heights when the other species is absent (Gross and Price 2000; Price and Gross 2005; see also Price 1981), indicating the converse of character displacement (character release) in the absence of one or the other species. Further supporting the role of competition, in south India, the association of tree height and tarsus length has evolved in parallel in a second species pair (Phylloscopus trochiloides with longer tarsus and higher foraging height; Phylloscopus occipitalis with shorter tarsus and lower foraging height). The south Indian pair is larger than the north Indian pair. These size differences are associated with the presence of more and larger food in the south, further evidence for a role of competition for food in driving patterns of coexistence and niche differentiation in the winter (Katti and Price 2003; Price and Gross 2005). Therefore, we will here consider character displacement to be an integral part of the production of species differences, associated with their coexistence in winter. This displace- 


\begin{tabular}{|c|c|c|}
\hline$t=0$ & WINTER & SUMMER \\
\hline & nonbreeding season & breeding season \\
\hline & Mortality & Reproduction \\
\hline & $\begin{array}{l}\text { Competition: } \\
\text { intra- and interspecific } \\
\text { phenotype-dependent }\end{array}$ & $\begin{array}{l}\text { Competition: } \\
\text { intraspecific } \\
\text { phenotype-independent }\end{array}$ \\
\hline & $\begin{array}{l}\text { Change in mean phenotype due to } \\
\text { selection }\end{array}$ & $\begin{array}{l}\text { Transmission of mean breeding value } \\
\text { across generations }\end{array}$ \\
\hline
\end{tabular}

Figure 2: Summary of processes occurring in the two seasons. Dotted lines indicate continuation from the previous summer and on to the following winter. The population is censused at the end of each winter.

ment could be considered to be both behavioral (tree height utilized) or morphological (tarsus length).

In the summer, all four species breed along the elevational gradient in the western Himalayas (Price 1991). The species with long tarsi (the small $P$. humei and large $P$. trochiloides) breed at higher elevations in birch forest, and the species with short tarsi (the small P. chloronotus and large $P$. occipitalis) breed at lower elevations in conifer. These summer habitat differences are again associated with foraging more efficiently by hopping at higher elevations or flying at lower elevations. The low and high species are altitudinal replacements of one another, coming into contact only at midelevations, where their habitats intergrade. However, the two higher-elevation species have vast ranges extending north into Siberia, whereas the lower-elevation species are confined to lower latitudes. It is plausible that competition between the altitudinal replacement species plays a role in mutually restricting each other's breeding ranges, but the majority of individuals of one species do not come into contact with individuals of the other. In the models we develop, we consider current competition between the species in the summer to be negligible. Breeding carrying capacities differ greatly, largely because a species at higher elevations has much more habitat to exploit than one at lower elevations. This means that for the same population sizes, the high-elevation species has many more resources available per individual. Not only are ranges larger, but arthropod abundance may increase to the north (Irwin 2000; see also Huston and Wolverton 2009), further increasing the total resource availability for the high-elevation species.

Here, we show how differences in total resource availability within the summer breeding ranges can result in asymmetrical evolution of the mean phenotypes of species (in the specific case above, this would be foraging height and tarsus length), which in turn affects winter abun- dances. Species with high breeding season output have a strong competitive effect on species with lower output, resulting in character displacement of the lower-output species to a less productive portion of the resource spectrum in winter, and hence lower population sizes. In this way, summer breeding conditions lead to perpetuation of population size differences through the annual cycle, even if density regulation is primarily a response to food shortages in winter.

In general terms, the questions we address are: What is the effect of differential population regulation in one season on character displacement in another? How does seasonal variation in interspecific competition affect the stability of coexistence between competitors? At the coevolutionary equilibrium, what are the competitive effects of one species on population size of the other?

\section{Model Description}

We build on existing models for the evolution of a quantitative trait that mediates competition between two species (Slatkin 1980; Taper and Case 1985; Case and Taper 2000). We allow, however, for separate dynamics in the winter or nonbreeding season and the summer or breeding season. The processes occurring in each season are summarized in figure 2. We are primarily concerned with effects of differential summer reproductive output on competitive interactions in the winter. Accordingly, we omit interspecific competition and selection in the summer and assume that evolution of the trait mediating winter competition is entirely determined by selection pressures in that season. We model differences in breeding output by assigning different summer carrying capacities to the species. The model tracks the abundance, $N_{i}(t)$, and mean phenotype, $\bar{z}_{i}(t)$, of each species $i$ over time $t$.

Throughout, parameters that apply within the winter 
and summer seasons are denoted with the superscripts $(w)$ and $(s)$, respectively. The durations of the two phases within each year are $T^{(w)}$ and $T^{(s)}$. A time unit of years is used, so $T^{(w)}+T^{(s)}=1$. The model description uses $t=0$ for the beginning of winter to keep the notation simpler, but the next winter starts at $t=1$, and so on. Within each season, dynamics are modeled in continuous time. This is natural to capture the density- and phenotype-dependent mortality occurring throughout the winter. It is less representative of a single summer breeding episode, but the important dynamic within that season is simply density-dependent reproduction, independent of phenotype, which can be represented equally well in continuous or discrete time. We further assume that generations are discrete and nonoverlapping. Each species is censused at the end of winter, when its abundance is at the lowest for the year.

\section{Winter}

During the winter, individuals compete with both conspecifics and heterospecifics for resources. The strength of this competition depends on phenotype, in two senses. In the relative sense, individuals that are more similar compete more strongly. In the absolute sense, the availability of resources follows a symmetric and peaked distribution, and competition is stronger among individuals with phenotypes best suited to exploit rarer resources.

Let $r_{i}^{(w)}(t, z)$ be the instantaneous growth rate (Malthusian fitness) for an individual of species $i$ with trait value $z$ at time $t$ during the winter:

$$
=r_{\max }^{(w)}\left(1-Q(z) \int_{-\infty}^{r_{i}^{(w)}(t, z)} \sum_{j} N_{j}(t) p_{j}\left(t, z^{\prime}\right) \alpha_{i j}\left(z-z^{\prime}\right) d z^{\prime}\right) .
$$

This is the continuous-time equivalent of Slatkin's (1980) equation (5). The index $j$ runs over all species, including $i$. The phenotype distribution of species $j$ is $p_{j}(t, z)$, which is assumed Gaussian with mean $\bar{z}_{j}(t)$ and constant variance $\sigma_{z}^{2}$. Our use of a constant phenotypic variance follows Case and Taper (2000) and presumes that genetic variance is maintained by a balance among mutation, recombination, and stabilizing selection (Lande 1975). The competition function is $\alpha_{i j}\left(z-z^{\prime}\right)=a_{i j} \exp \left[-\left(z-z^{\prime}\right)^{2} /\left(4 \sigma_{u}^{2}\right)\right]$. It is formed from the overlapping Gaussian resource utilization functions, each with width $\sigma_{u}$ (Roughgarden 1979, p. 519), of two individuals of phenotypes $z$ and $z^{\prime}$. The constant $a_{i j}$ allows for species-specific, phenotype-independent asymmetric competition, scaling the effect of species $j$ on $i$. The same-species $a$ 's, $a_{i i}$, will be kept equal to 1 , but the cross-species $a$ 's may take nonnegative values greater or less than 1.

There is only mortality, not reproduction, during the winter, so $r_{\max }^{(w)}$ is negative. The function $Q(z)$ describes density dependence, and we assign it to be

$$
Q(z)=-\frac{1}{Q_{0}} \exp \left(\frac{z^{2}}{2 \sigma_{\mathrm{Q}}^{2}}\right)
$$

to reflect the consequences of a Gaussian form of resource availability on the number of individuals that can be supported (Roughgarden 1976; Slatkin 1980). Stabilizing selection from the resource distribution itself is thus about an optimum phenotype of $z=0$, which does not vary with time. The quantity $Q_{0}$ mediates density dependence in a manner analogous to carrying capacity, so that the per capita mortality rate (eq. [1]) increases with increasing abundance. It is not carrying capacity in the usual sense, however, because the equilibrium population sizes would be zero if these mortality-only winter dynamics continued indefinitely (Gabriel et al. 2005).

The mean fitness of species $i$ during the winter is

$$
\bar{r}_{i}^{(w)}(t)=\int_{-\infty}^{\infty} p_{i}(t, z) r_{i}^{(w)}(t, z) d z .
$$

Changes in population sizes and mean phenotypes over the winter $\left(0<t \leq T^{(w)}\right)$ are described by Lande $(1982)$ :

$$
\begin{aligned}
\frac{d N_{i}}{d t} & =\bar{r}_{i}^{(w)}(t) N_{i}(t), \\
\frac{d \bar{z}_{i}}{d t} & =\sigma_{z}^{2} \frac{\partial \bar{r}_{i}^{(w)}(t)}{\partial \bar{z}_{i}} \\
& =\int_{-\infty}^{\infty} z p_{i}(t, z) r_{i}^{(w)}(t, z) d z-\bar{z}_{i} \bar{r}_{i}^{(w)}(t) .
\end{aligned}
$$

There is no frequency-dependent term in equation (5a) because the competition function and phenotype distribution are both symmetric in $z$ (Case and Taper 2000). We omit division by the generation time in equation (5) because it is assumed to be one, so as to avoid complications arising from age structure. Heritability does not enter equation (5) because transmission across generations occurs at the end of the summer (eq. [9]). Because equation (5) employs the selection gradient in continuous time and assumes that the phenotype distributions remain Gaussian, it supposes that selection is relatively weak.

The Gaussian forms of $p_{i}, \alpha_{i j}$, and $1 / Q(z)$ permit simplification of equations (1), (3), and (5); results are given in appendix A. Further discussion of the model of winter 
character displacement, including conditions for character convergence and extinction, appears in appendix $\mathrm{B}$.

To find the abundances and mean phenotypes at the end of the winter, we step through equations (4) and (5) (hence recalculating eqq. [1] and [3]) with small values of $d t$ over the time period $T^{(w)}$. The values at the end of the winter, $N_{i}\left(T^{(w)}\right)$ and $\bar{z}_{i}\left(T^{(w)}\right)$, become the initial conditions for the summer phase.

\section{Summer}

During the summer, only intraspecific competition affects each species. We assume that all individuals within a species are equal competitors in this season, so that reproductive success does not depend on the phenotype being modeled, or on an individual's condition after surviving the winter. The population size of each species grows logistically, with carrying capacity $K_{i}^{(s)}$ and intrinsic rate of growth $r_{\max }^{(s)}$, for duration $T^{(s)}$. Let $r_{i}^{(s)}(t)$ be the instantaneous growth rate (Malthusian fitness) for an individual of species $i$ at time $t$ during the summer. Because we assume that growth rate does not depend on individual phenotype $z$, the mean fitness of the species, $\bar{r}_{i}^{(s)}(t)$, is identical to the individual fitness (growth rate):

$$
\bar{r}_{i}^{(s)}(t)=r_{i}^{(s)}(t)=r_{\max }^{(s)}\left(1-N_{i}(t) / K_{i}^{(s)}\right) .
$$

Changes in population size over the summer $\left(T^{(w)}<\right.$ $t \leq T^{(w)}+T^{(s)}$ ) are then described by

$$
\frac{d N_{i}}{d t}=\bar{r}_{i}^{(s)}(t) N_{i}(t)
$$

The abundances at the end of the summer are therefore

$$
N_{i}\left(T^{(s)}\right)=\frac{N_{i}(0) K_{i}^{(s)}}{N_{i}(0)+\left[K_{i}^{(s)}-N_{i}(0)\right] \exp \left[-r_{\max }^{(s)} T^{(s)}\right]} .
$$

The mean phenotype of each species does not change over the summer. The preceding winter's selection, however, causes changes in the mean phenotype of the offspring generation with respect to the parents. For a single character and nonoverlapping generations, the breeder's equation (Falconer and McKay 1996, p. 186) summarizes phenotypic change from one year to the next. The mean phenotype of those that survived the winter and can breed in the summer (i.e., after selection but before reproduction) is $\bar{z}_{i}\left(T^{(w)}\right)$. The mean phenotype at the beginning of the preceding winter is $\bar{z}_{i}(0)$. With $h^{2}$ denoting heritability and a generation time of 1 year, the mean phenotype at the end of the summer is thus

$$
\bar{z}_{i}(1)=\bar{z}_{i}(0)+h^{2}\left[\bar{z}_{i}\left(T^{(w)}\right)-\bar{z}_{i}(0)\right] .
$$

The population sizes and mean phenotypes at the end of the summer (eqq. [8], [9]) become the new values for the beginning of the winter in the next year.

\section{Model Analysis}

We start by illustrating the general behavior of the model (fig. 1) before considering detailed results. A single species exploiting a symmetrical distribution of resources has its equilibrium mean phenotype at the value corresponding to the peak of the resource distribution (fig. 1A). When two equivalent species are present, both can persist with equal abundances and mean phenotypes symmetrically displaced from the optimum (fig. 1B). Each species' mean phenotype is under stabilizing selection: those individuals near the center of the resource distribution suffer intense interspecific competition, whereas those individuals on the outer extremes encounter few resources.

Now suppose that species 1 has more extensive summer breeding grounds and hence a larger summer carrying capacity, $K_{1}^{(s)}>K_{2}^{(s)}$. The consequent higher return of species 1 reduces the fitness of individuals of species 2 in the left tail, resulting in directional selection on this species, causing it to evolve toward the right, farther from the optimum. Furthermore, as species 2 moves right, species 1 is also under directional selection to take advantage of the center of the resource distribution. A new equilibrium is reached where species 1 exploits most of the resources and species 2 is displaced farther into the tail of the resource distribution (fig. 1C).

\section{Progress to Equilibrium}

We now consider the dynamics of population size and mean phenotype that lead to the asymmetric outcome just described (fig. 1C). Even if species 1 has an initial disadvantage in both abundance and phenotype, its summer advantage allows it to become more abundant in the winter (fig. $3 A$ ), with a mean phenotype closer to the optimum than that of species 2 (fig. $3 B$ ). In this example, the abundance dynamics show a rapid decline followed by a slow rise. Species 2 also shows a later decline in abundance as its phenotype is pushed farther from the optimum. With different initial conditions, the transient dynamics are different (though still not complex), but the equilibrium outcome is the same.

In figure 4, we show the complete phenotypic distributions and winter fitness functions experienced by each species during the progress to equilibrium. Over time, the mean phenotype of each species moves toward values conferring higher (less negative) winter fitness, simultaneously changing the fitness surfaces themselves (fig. 4A-4C). The equilibrium values of $\bar{z}_{i}$ are not quite at the mean fitness peaks (fig. 4C) because of the separate summer dynamics. 

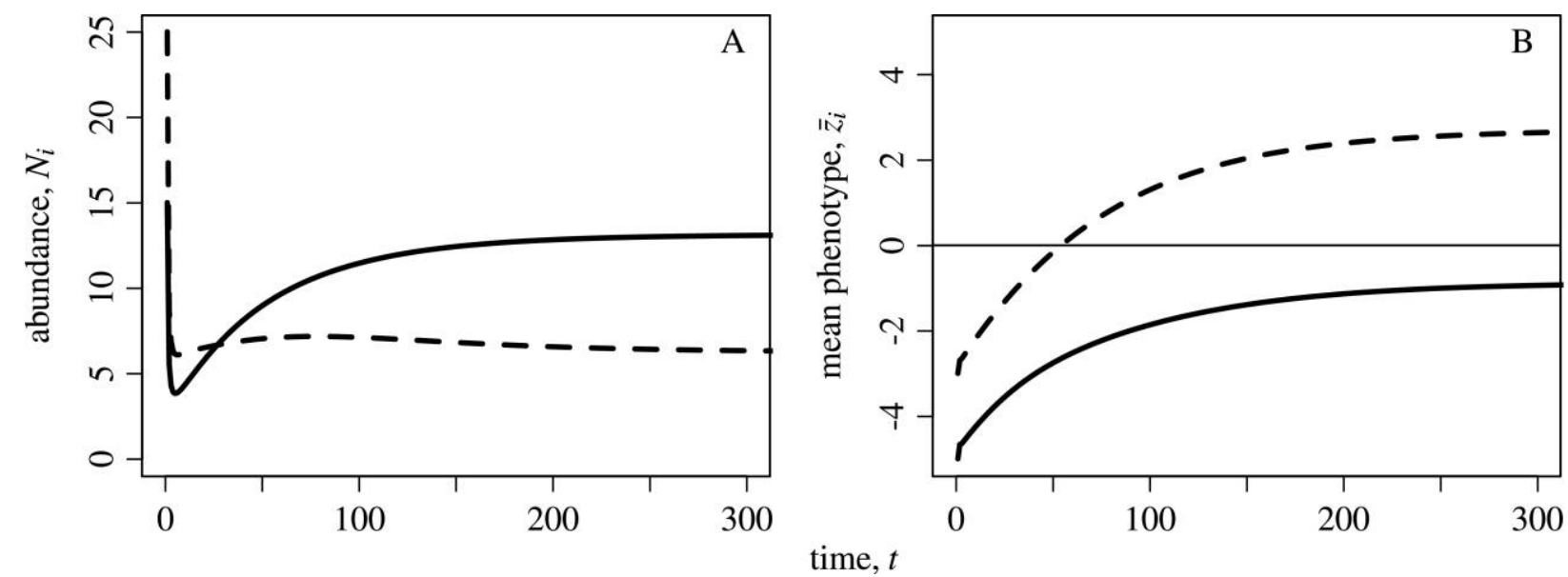

Figure 3: Time development of the seasonal coevolutionary system, toward the equilibrium shown in figure $1 C$. The abundances $(A)$ and mean phenotypes $(B)$ for species 1 and 2 are shown by the solid and dashed lines, respectively. Species 1 starts with a smaller population size and a mean phenotype farther from the optimum (which is $z=0$, shown by the thin line) than species 2 . Parameter values are as in figure 1.

At this equilibrium, the more abundant species 1 claims the region of optimum phenotype, and species 2 has local maxima in mean fitness at both much smaller and much greater phenotype values. The more abundant species has a lower equilibrium mean fitness in the winter than the less abundant species does because of greater intraspecific competition induced by its higher mean fitness in the summer. This occurs despite its mean phenotype being closer to the winter optimum (fig. $4 F$ ).

\section{Coexistence}

Species are likely to have competitive effects on each other in ways beyond those mediated by the single trait thus far considered. Here we consider first the situation in which the species' competitive strengths are asymmetric, so that one species has a stronger effect on the other. Then we consider situations in which the species are symmetric but in which the strength of competition between two individuals depends not only on their phenotypes but also on whether they are conspecifics or heterospecifics. We examine the consequences of these forms of phenotypeindependent interspecific and intraspecific competition on the coexistence of the two competitors. The conditions for coexistence versus competitive exclusion in the absence of seasonal effects are summarized in appendix B.

Asymmetric Competition. For the warblers on which our model is based, the species with a breeding range that extends into Siberia has a high summer carrying capacity but also a longer migration distance. Potentially, this could reduce its competitive ability in the winter, for example, because individuals breeding closer to the winter grounds can establish winter territories more quickly, or because the rigors of migration lower body condition. We are hence interested in how summer breeding productivity and winter competitive ability interact.

The consequences of higher summer carrying capacity for a weaker winter competitor are illustrated in figure 5. The mean phenotype of the inferior competitor (species 1) improves as its summer advantage increases. The stronger competitor (species 2) claims a mean phenotype much closer to the optimum, however, even when the abundance of species 1 is high. An advantage in summer carrying capacity can thus offset weaker competitive strength in the winter, at least in abundance if not in phenotype. The scenario shown in figure 5 produces the surprising outcome that the more abundant species may be the one with mean phenotype farther from the winter optimum.

A high summer carrying capacity can even save an inferior competitor from winter-driven extinction. To show this, we narrow the resource distribution so that the strength of stabilizing selection is sufficient to prevent character displacement. In this case, without the separate breeding season, the worse competitor (species 1) would become extinct, as discussed in appendix B. But with the summer dynamics species 1 can persist, even if the carrying capacities of the two species in the summer are identical. Continuing with the example from figure 5 but reducing the width of the stabilizing selection function attributed to resources, $\sigma_{Q}^{2}=2.25$ (cf. 4.0), when $K_{1}^{(s)}=K_{2}^{(s)}=20$, the weaker competitor persists at about half the abundance 

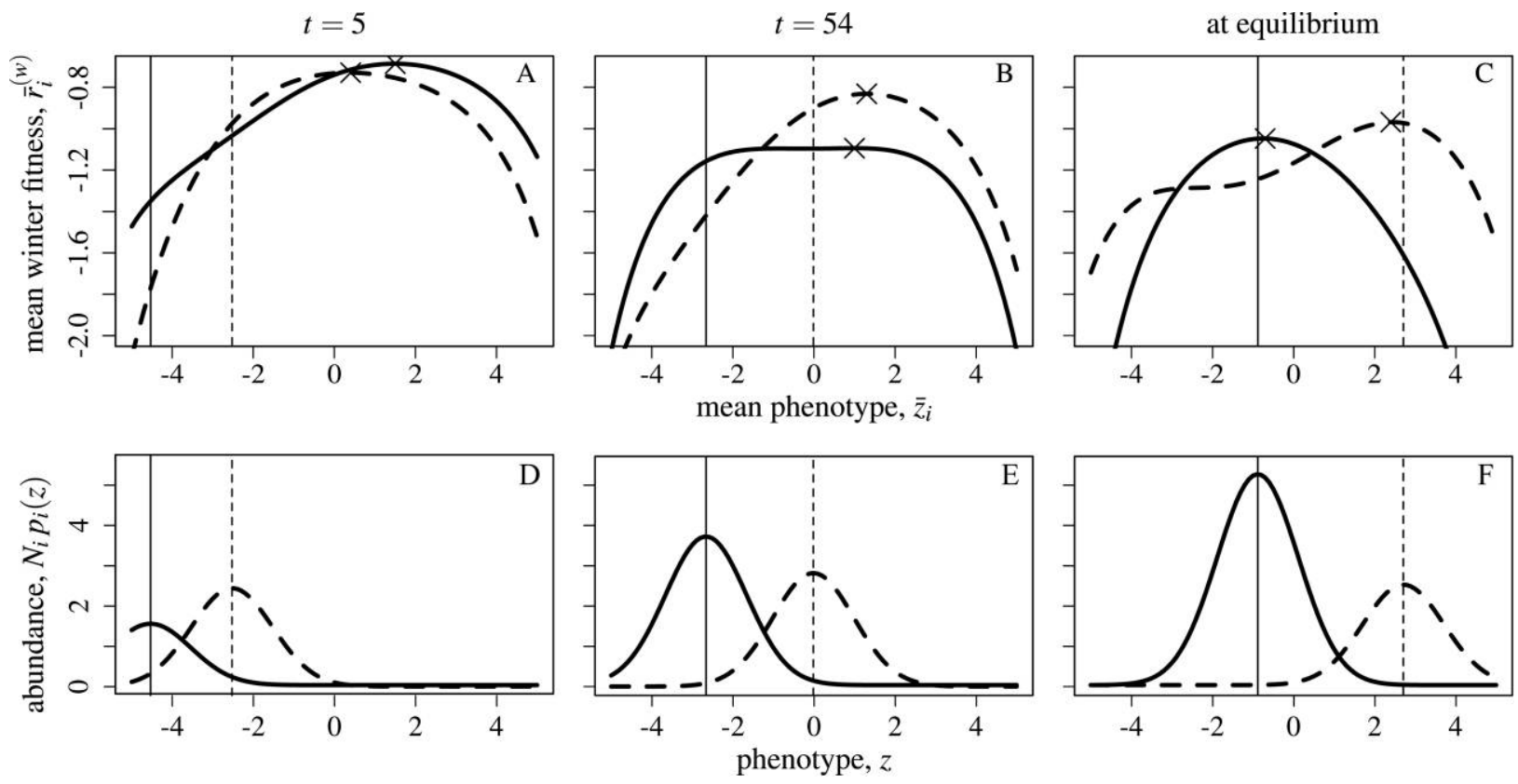

Figure 4: Mean fitness functions $(A-C)$ and their corresponding phenotype distributions $(D-F)$ in three different years $(t)$ during the approach to equilibrium illustrated in figure 3 . The thin vertical lines mark the mean phenotypes of each species (solid for $\bar{z}_{1}$, dashed for $\bar{z}_{2}$ ). A-C show $\bar{r}_{i}^{(w)}$ (eqq. [3] and [A3]) evaluated at $\bar{z}_{j}$ for a range of values of $\bar{z}_{i}$ (solid lines for $i=1, j=2$; dashed lines for $i=2, j=$ 1). Cross symbols in these panels mark the maximum value of each mean fitness function.

of species 2, and with a mean phenotype nearly as close to the optimum. The summer advantage required for $N_{1}$ to exceed $N_{2}$ is a bit more than in figure 5 , around $K_{1}^{(s)}=100$, but it is possible, and again both mean phenotypes are quite close to zero with little character displacement.

The seasonality of this system, and in particular the separate summer reproductive or recovery period, therefore rescues weaker competitors in two ways. First, the summer recovery period allows a species to attain high abundance even when phenotypically displaced from the winter optimum (fig. 5), and, second, the summer recovery period enables winter coexistence when the outcome would otherwise be competitive exclusion.

Symmetric Competition. We now consider species that have symmetric effects on each other, but for which the strengths of interspecific and intraspecific competition inherently differ, again independent of the phenotype modeled, that is, $a_{12}=a_{21} \neq 1$. For example, the species may deplete other resources at different rates or share predators (e.g., Martin and Martin 2001). Or, competitive interactions may be fundamentally different within species than between species (neighbor-dependent selection; Vasseur et al. 2011).
In figure 6, we show how the strength of phenotypeindependent interspecific competition interacts with summer carrying capacity differences to determine equilibrium abundances and mean phenotypes. When the two species are symmetric in every respect, increasing interspecific competition results in decreased population sizes of each, and their greater displacement from the optimum, as expected. When the summer carrying capacity of species 1 is several times higher than that of species 2 , increasing interspecific competition has only a small effect on the population size of the more abundant species. This is because species 1 is limited much more by intraspecific than by interspecific competition. Strong interspecific competition does, however, allow species 1 to evolve closer to the resource optimum because it greatly decreases the abundance of species 2 . On the other hand, the numerical dominance of species 1 means that the species with low summer carrying capacity is increasingly affected as the strength of interspecific winter competition increases. In the severe case of a 10-fold difference in summer carrying capacities and competition 1.75 times stronger between heterospecifics than conspecifics, the equilibrium winter population size of species 2 is reduced eightfold and its phenotype distribution is displaced more than four standard deviations from the optimum. 


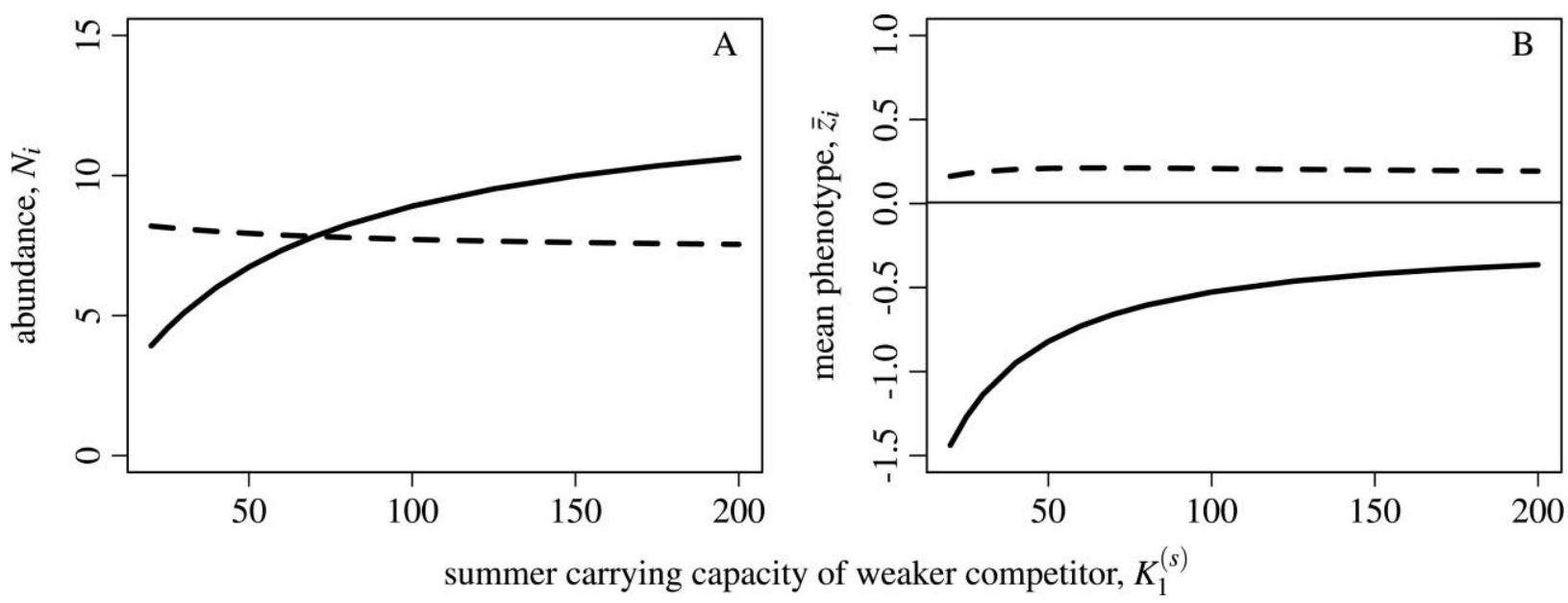

Figure 5: Equilibrium abundance $(A)$ and mean phenotype $(B)$ of the weaker (species 1, solid lines) and stronger (species 2, dashed lines) competitors, as a function of summer carrying capacity of species 1 . Parameter values are as in figures $1-4$, except $\sigma_{Q}^{2}=4, a_{12}=1.8$, and $a_{21}=0.2$. The leftmost points are for $K_{1}^{(s)}=20=K_{2}^{(s)}$, when neither species has a summer advantage.

\section{Ecological and Evolutionary Release}

All results described above for equilibrium population sizes (e.g., figs. 1, 5, 6) reflect both the immediate effects of one competitor on the other and the consequences of evolutionary changes. To compare these "ecological" and "evolutionary" contributions, we start with the two species at their coevolutionary equilibrium and remove species 2 . We then track the population size of species 1, which initially has its equilibrium abundance $N_{1}^{*}$ and mean phenotype $\bar{z}_{1}^{*}$. First, we prohibit the evolution of $\bar{z}_{1}$ by setting $h^{2}=0$; reducing the heritability reduces the speed of evolutionary response (to zero, in our case; see also Schreiber et al. 2011; Vasseur et al. 2011). We then observe the new equilibrium abundance $N_{1}^{\prime}$ and mean phenotype $\bar{z}_{1}^{\prime}$, which are reached in only a few generations. These changes in abundance, $\Delta N_{\text {есо }}=N_{1}^{\prime}-N_{1}^{*}$, and mean phenotype, $\Delta \bar{z}_{\text {eco }}=\bar{z}_{1}-\bar{z}_{1}^{*}$, are the "ecological" response. The change in mean phenotype is small, only because of phenotypedependent winter mortality. Second, we restore inheritance (to any value of $0<h^{2} \leq 1$ ) and let the full evolutionary dynamics play out so that species 1 goes to the single species equilibrium $\bar{z}_{1}^{\prime \prime}=0$. The equilibrium abundance $N_{1}^{\prime \prime}$ under these conditions includes both the ecological and evolutionary responses. The abundance difference $\Delta N_{\text {evo }}=N_{1}^{\prime \prime}-N_{1}^{\prime}$ and mean phenotype difference $\Delta \bar{z}_{\text {evo }}=\bar{z}_{1}^{\prime \prime}-\bar{z}_{1}$ are the evolutionary response.

Results are illustrated in figure 7. When $K_{1}^{(s)} \simeq K_{2}^{(s)}$, the magnitudes of the ecological and evolutionary abundance responses are comparable. When species 1 has higher summer population size, $K_{1}^{(s)} \gg K_{2}^{(s)}, \Delta N_{\text {eco }}$ remains stable but $\Delta N_{\text {evo }}$ is reduced because there was less character displace- ment from which to recover; $\Delta \bar{z}_{\text {evo }}$ is thus also smaller. Repeating this exercise but keeping species 2 as the survivor, we again find comparable magnitudes of the ecological and evolutionary changes in abundance (results not shown). In this case, however, both of these responses increase with $K_{1}^{(s)}$ (now the summer carrying capacity of the removed species) because the surviving species was more greatly displaced (cf. fig. 1C).

\section{Discussion}

When species compete, evolutionary outcomes depend on their abundances and on the degree to which they exploit common resources. We have shown how differences in population sizes resulting from differences in total resource availability in one season (here, the summer or breeding season) strongly affect evolutionary outcomes determined by competitive interactions in the other season (here, the winter or nonbreeding season). In order to dissect the cross-seasonal effect of reproductive output on evolution, we have assumed that trait-mediated competition occurs only in the winter. Our equations thus encompass situations where each species is independently regulated in the summer. Although competition between species in the summer is not explicitly modeled and cannot evolve, the difference in summer carrying capacities could be viewed as its outcome.

In general, evolutionary change in one species affects population sizes of that species and all those with which it interacts (Slatkin 1980; Hairston et al. 2005; Schoener 2011; Vasseur et al. 2011). First, with respect to the focal 

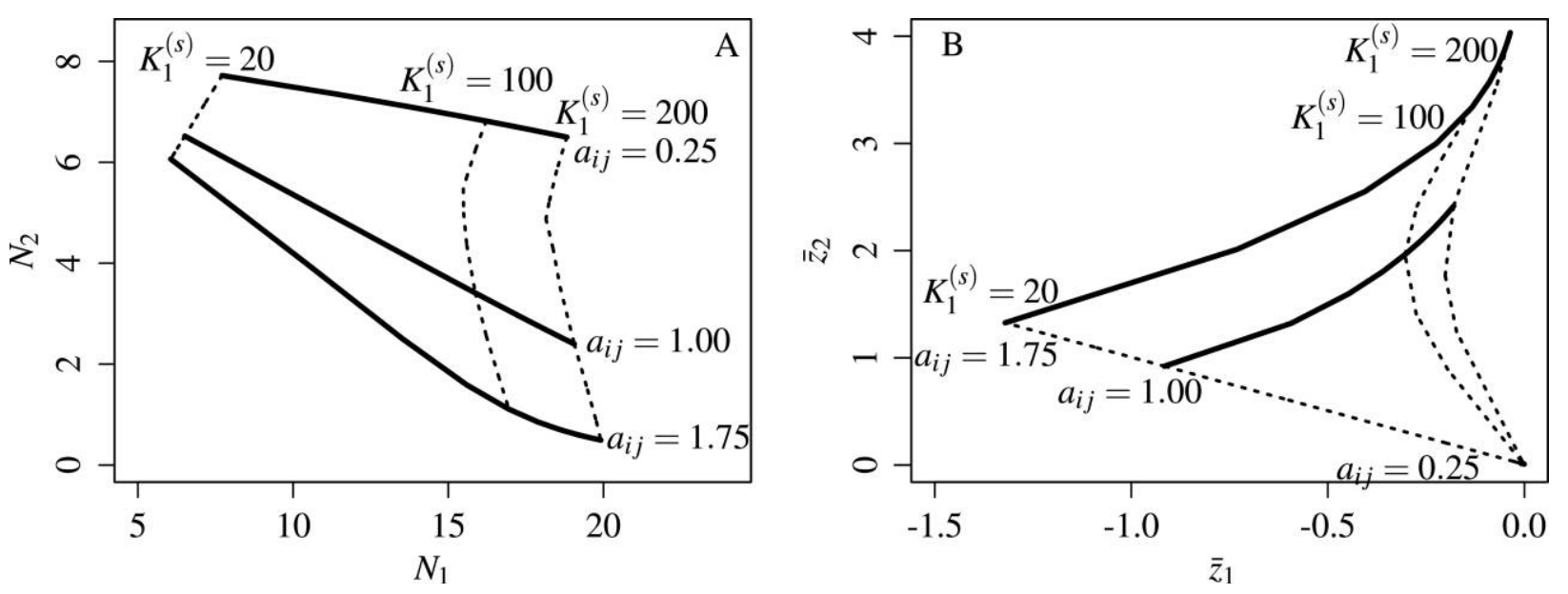

Figure 6: Effects of phenotype-independent interspecific competition and summer carrying capacity on equilibrium abundance $(A)$ and mean phenotype $(B)$. The three thick lines show results for different strengths of interspecific competition, for the value of $a_{i j}=a_{12}=$ $a_{21}$ indicated. The dotted lines show results for values of $K_{1}^{(s)}$ increasing to the right from 20 to 100 to 200 . The leftmost dotted line in $A$ and bottommost dotted line in $B$ thus represent the situation where the species are symmetric in every respect. Other parameter values are as in figure 5 .

species, adaptation to the environment (in our case, including the abundance and phenotype of a competitor) raises population sizes to levels higher than expected in the absence of evolution (Hairston et al. 2005; Ellner et al. 2011). Second, adaptation can have ramifying effects through the food web (Hairston et al. 2005; Schoener 2011). In some models evolution can result in cycling population sizes and even be essential to the maintenance of coexisting species (Schreiber et al. 2011; Vasseur et al. 2011). In these cases, the speed of evolutionary response, as determined by the product of selection and heritability, is critical to the outcome. In our system, heritability does not affect whether a pair of competing species can coexist, and it has only a very small effect on their equilibrium abundances and phenotypes ( $\lesssim 1 \%$; results not shown), but the equilibrium is reached more slowly with lower heritabilities. We found that a typical outcome of removing a competitor is that the immediate (ecological) increase in population size was roughly the same as the increase resulting from subsequent evolution over several tens of generations (fig. 7). The order-of-magnitude similarity of the evolutionary and ecological responses across generations is in accord with some empirical studies, which have demonstrated that strong selection and accompanying evolution may rapidly restore a depressed population size in a changing environment (Hairston et al. 2005; Ellner et al. 2011).

The consequence of differential reproductive output in one season on evolution in the other also depends on competitive effects that are not mediated by phenotype.
These effects are magnified as interspecific competition increases relative to intraspecific competition (fig. 6). Differences between seasons also mean that a poorer competitor in one season need not be driven rare or extinct if it has sufficiently high productivity in the other season. An inferior competitor with high carrying capacity in the breeding season undergoes rapid growth from low population sizes each year. This provides not only a direct subsidy to its abundance, but the increased intraspecific competition drives its mean phenotype to evolve closer to the optimum, thereby reducing the severity of winter resource limitation for that species. All of this may happen with only a small effect on the superior competitor (fig. 5).

Our results show that populations that are strongly regulated by food in the winter may nevertheless have their abundances determined by conditions in the summer, as a result of evolutionary adjustments. They explain the apparently paradoxical finding of exactly this pattern in the Old World leaf warblers (Price and Gross 2005), as described in the "Introduction." The approach also applies generally to species that encounter different competitive interactions and resource distributions at different times of the annual cycle-perhaps the majority of the world's species. The model can be expanded to accommodate more species and also situations in which multiple traits mediate competitive interactions at multiple points in the annual life cycle. In these cases, we expect abundances and phenotypes to evolve as a compromise between any op- 

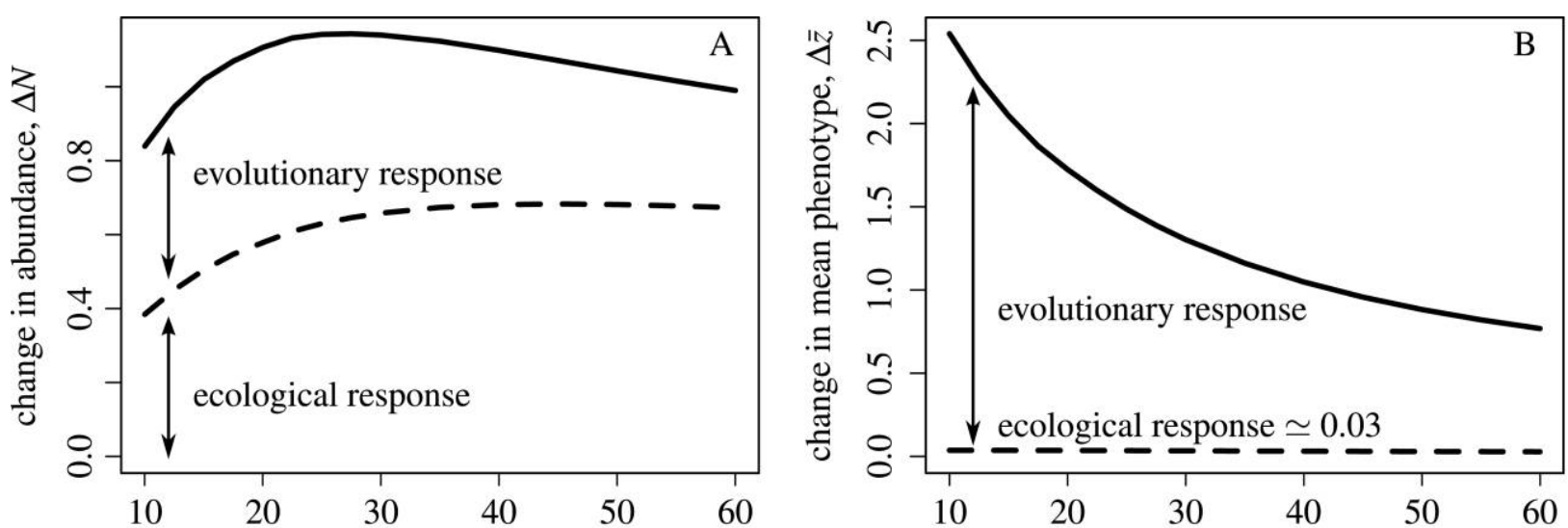

summer carrying capacity of surviving species, $K_{1}^{(s)}$

Figure 7: Ecological and evolutionary components of competitive release for abundance $(A)$ and mean phenotype $(B)$, as functions of summer carrying capacity differences. Solid and dashed lines show the change in species 1 after species 2 is removed, with and without inheritance, respectively. Thus, the dashed lines show the ecological response, $\Delta N_{\text {eco }}$ and $\Delta \bar{z}_{\text {eco }}$, and the distance between the dashed and solid lines is the evolutionary response, $\Delta N_{\text {evo }}$ and $\Delta \bar{z}_{\text {evo }}$. Parameter values are as for figure 1, except for the summer carrying capacity of species 1 and the heritability ( $h^{2}=0.5$ for the initial coevolutionary equilibrium, but $h^{2}=0$ for the dashed lines).

posing selection pressures that develop as a result of differences in resource distributions and population sizes.

Here, we have considered that competitive interactions are mediated by a unimodal, continuous resource distribution. Although not empirically assessed, this may apply to many aspects of warbler biology, such as distributions along a foraging height gradient. However, we have not developed models of multimodal resource distributions, and these may lead to different outcomes. For example, food limitation in Darwin's ground finches probably plays a major role in regulating population sizes (Grant and Grant 1996), just as inferred for the Old World warblers. However, in the finches such limitation is accompanied by strong selection to efficiently exploit specific seeds (Grant and Grant 2002, 2006), and the adaptive surface set by these discrete resources has tall peaks and deep valleys (Schluter and Grant 1984; Schluter et al. 1985). In the ground finches, the power of differential productivity in the breeding season to drive species off their nonbreeding season resource peak is likely to be relatively small, given the strong selection to efficiently utilize the available food supply in that season.

Within-season temporal variation in the resource distribution could also be considered in this framework. Simple resource changes may not greatly alter our conclusions. For example, narrowing of the resource distribution as the winter progresses might have a net effect similar to the model with a constant but somewhat smaller value of $\sigma_{Q}$. More complex changes in the resource distribution, especially those driven by resource use of the competing species themselves, may, however, have substantially different consequences on character displacement and coexistence.

Cross-seasonal implications of population regulation are not restricted to summer effects on winter evolution. For example, differential survival in the nonbreeding season will affect the outcome of competitive interactions in the breeding season. Martin and Martin (2001) experimentally demonstrated strong breeding season competition between two New World warblers, Vermivora celata and Vermivora virginiae, with V. celata dominant. Vermivora celata is much more abundant and occupies a much larger winter range, implying that difference in winter abundance is a possible factor driving evolution of summer competitive asymmetry. More generally, in the New World warblers, observational and experimental data indicate density-dependent regulation, via food, is operating in both the summer and winter (Rodenhouse et al. 2003; Sillett et al. 2004; Faaborg et al. 2010). Bassar et al. (2010) have identified several other experiments across a variety of taxa, demonstrating that density dependence often operates at multiple stages of the life history. These findings are in accord with our results, which have shown how competition through food limitation in one season can affect population dynamics in the other season, rather than either season dominating the regulatory process. Although we did not explore it here, censusing populations at multiple stages in their life cycle (e.g., end of summer as well as end of winter) could shed additional light on the differ- 
ential seasonal contributions to population dynamics and trait evolution.

When species interact, it has long been recognized that evolutionary change in one species alters the environment experienced by another, resulting in evolution of the second species and the potential for mutual feedbacks (Post and Palkovacs 2009; Bolnick et al. 2011; Vasseur et al. 2011). Here we have shown that in competitive systems, even changing the abundance of one species can have profound effects on rates and directions of evolution of the other. Much has been made of how human impacts directly place new selection pressures on populations (Palumbi 2001), and even more about how human impacts are altering population sizes, decreasing the abundance of many species, and increasing the abundance of a few (Butchart et al. 2010). The findings reported here link these two consequences of human activities by showing how changes in population sizes lead to large evolutionary adjustments; the net result may be further changes in population sizes due to evolution, over and above those predicted from analyses based on community ecology.

\section{Acknowledgments}

This article benefited from the comments of two anonymous reviewers. Work was supported by National Science Foundation grants DEB-0919089 and DEB-1120279 (E.E.G.) and a Royal Society Research Professorship (R.L.).

\section{APPENDIX A}

\section{Winter Coevolution Functions}

The Gaussian forms of $p_{i}(z), \alpha_{i j}\left(z-z^{\prime}\right)$, and $1 / Q(z)$ permit simplification of equations (1), (3), and (5).

Define the following quantities to simplify notation:

$$
\begin{aligned}
\sigma_{u z}^{2} & =2 \sigma_{u}^{2}+\sigma_{z}^{2}, \\
A & =\frac{1}{2 \sigma_{Q}^{2} \sigma_{u z}^{2} \sigma_{z}^{2}}, \\
b & =\sigma_{Q}^{2} \sigma_{z}^{2}+\sigma_{Q}^{2} \sigma_{u z}^{2}-\sigma_{z}^{2} \sigma_{u z}^{2}, \\
c & =\bar{z}_{i} \sigma_{Q}^{2} \sigma_{u z}^{2}+\bar{z}_{j} \sigma_{Q}^{2} \sigma_{z}^{2}, \\
d & =\bar{z}_{i}^{2} \sigma_{Q}^{2} \sigma_{u z}^{2}+\bar{z}_{j}^{2} \sigma_{Q}^{2} \sigma_{z}^{2} .
\end{aligned}
$$

Individual winter fitness (eq. [1]), mean winter fitness (eq. [3]), and the winter selection gradient (used in eq. [5]) then become

$$
\begin{gathered}
r_{i}^{(w)}(t, z) \\
=r_{\max }^{(w)}\left\{1+\frac{\sqrt{2} \sigma_{u}}{Q_{0} \sigma_{u z}} \exp \left[\frac{z^{2}}{2 \sigma_{Q}^{2}}\right] \sum_{j} a_{i j} N_{j} \exp \left[\frac{-\left(z-\bar{z}_{j}\right)^{2}}{2 \sigma_{u z}^{2}}\right]\right\}, \\
=r_{\max }^{(w)}\left\{1+\frac{1}{Q_{0}} \sqrt{\frac{2}{b}} \frac{\sigma_{u} \sigma_{Q}}{\sigma_{z}^{2}} \sum_{j} a_{i j} N_{j} \exp \left[-A\left(d-\frac{c^{2}}{b}\right)\right]\right\}, \\
=\frac{\partial \bar{r}_{i}^{(w)}(t)}{\partial \bar{z}_{i}} \\
r_{\max }^{(w)} \sqrt{\frac{2}{b}} \frac{\sigma_{u} \sigma_{Q}}{\sigma_{z}^{2}} \sum_{j} a_{i j} N_{j}\left(\frac{c}{b}-\bar{z}_{i}\right) \exp \left[-A\left(d-\frac{c^{2}}{b}\right)\right],
\end{gathered}
$$

provided that $b>0$ for convergence of the integral in equation (3). A sufficient but not necessary condition for $b>0$ is $\sigma_{Q}^{2}>\sigma_{z}^{2}$.

\section{APPENDIX B}

\section{Winter Character Displacement Conditions}

To understand the model of competitive dynamics, we set aside the effects of seasonality for the moment and focus on the phenotype-dependent competition described in equation (1). Specifically, for this appendix only, we set $T^{(s)}=0$ and $T^{(w)}=1$, assume $r_{\max }^{(w)}>0$, and remove the leading negative sign from $Q(z)$ in equation (2). We also insert a factor of $h^{2}$ in equation (5), although this does not affect the results unless the two species have different heritabilities for the trait in question. This excursion provides a baseline against which the effects of the separate breeding season can be measured and informs the parameter values we use for the full seasonal analyses.

Our model shares a framework commonly employed for studying ecological character displacement of a quantitative trait. It uses Slatkin's (1980) extension of Roughgarden's (1972) formulation (see also Taper and Case 1985) to describe the strength of competition in terms of differences in phenotype value. Stabilizing selection is density dependent because it enters through the carrying capacity function, as in Slatkin (1980) and Taper and Case (1985). This is in contrast to the treatments of Case and Taper (2000) and Goldberg and Lande (2006), which impose stabilizing selection through an additional densityindependent term in the individual fitness function, and of Price and Kirkpatrick (2009), in which stabilizing selection enters as an adjustment to $r_{\max }$. We also assume that the phenotypic variance of each species returns to a constant value at the beginning of each generation (following Case and Taper 2000; Goldberg and Lande 2006; in contrast to Taper and Case 1985; Slatkin 1980). This assumption is based on a balance of stabilizing selection 
versus mutation and recombination in maintaining heritable variability in a polygenic trait (Lande 1975).

Character convergence occurs when the maximum fitness of a rare invader (species 2) to a single-species system at equilibrium $\left(\bar{z}_{1}=0\right)$ is at the optimum phenotype, $\bar{z}_{2}=0$ (Case and Taper 2000). From equation (A3), stabilizing selection is sufficiently strong to force convergence when $\sigma_{Q}^{2}<2 \sigma_{u}^{2}+\sigma_{z}^{2}$, in agreement with Taper and Case (1985). When this condition is true, the $a_{i j}$ determine extinction or coexistence exactly as they do in nonevolutionary Lotka-Volterra dynamics (e.g., MacArthur and Levins 1967). Assuming equal carrying capacities for the two species and $a_{11}=a_{22}=1$, species 1 becomes extinct if $a_{12}>1$ and $a_{21}<1$, or if it has an initial disadvantage, $a_{12}>1$, and $a_{21}>1$. When $a_{i j}=1$, the abundances of the two species are neutrally stable. (As we show in the main text, however, including the summer dynamics causes the character convergence outcome to become locally stable by allowing the species with lower abundance to catch up by reproduction.)

Alternatively, when $\sigma_{Q}^{2}>2 \sigma_{u}^{2}+\sigma_{z}^{2}$, stabilizing selection is weak enough that character displacement occurs. Competition will not drive one species extinct in this case, even for extreme values of the $a_{i j}$. This result differs from Slatkin's (1980) result because of the differing assumptions about the phenotypic variance. When the phenotypic variance of the more abundant species is allowed to increase, it leaves less room for the displaced species, driving it to extinction. In our model, however, phenotypic variancer are fixed and the carrying capacity never falls completely to zero. The displaced species can therefore always evolve an extreme enough phenotype to persist, at least at low abundance.

In terms of the Lotka-Volterra conditions, this guaranteed coexistence may be understood by computing the average effect, across the entire phenotype distributions of both species, of one species on the other, $\mathbf{A}\left(\bar{z}_{1}-\bar{z}_{2}\right)$ :

$$
\begin{aligned}
\mathbf{A}\left(\bar{z}_{1}-\bar{z}_{2}\right)= & \int_{-\infty}^{\infty} \int_{-\infty}^{\infty} \alpha_{12}\left(z-z^{\prime}\right) p_{1}\left(z, \bar{z}_{1}\right) \\
& \times p_{2}\left(z^{\prime}, \bar{z}_{2}\right) d z d z^{\prime} \\
= & a_{12} \sqrt{\frac{\sigma_{u}^{2}}{\sigma_{u}^{2}+\sigma_{z}^{2}}} \exp \left[\frac{-\left(\bar{z}_{1}-\bar{z}_{2}\right)^{2}}{4\left(\sigma_{u}^{2}+\sigma_{z}^{2}\right)}\right] .
\end{aligned}
$$

The total effect of species 2 on species 1 therefore becomes small when the mean phenotypes of the species diverge (fig. B1). Consequently, character displacement en sures that the overall competition coefficients remain below 1, allowing coexistence even of a much weaker competitor.

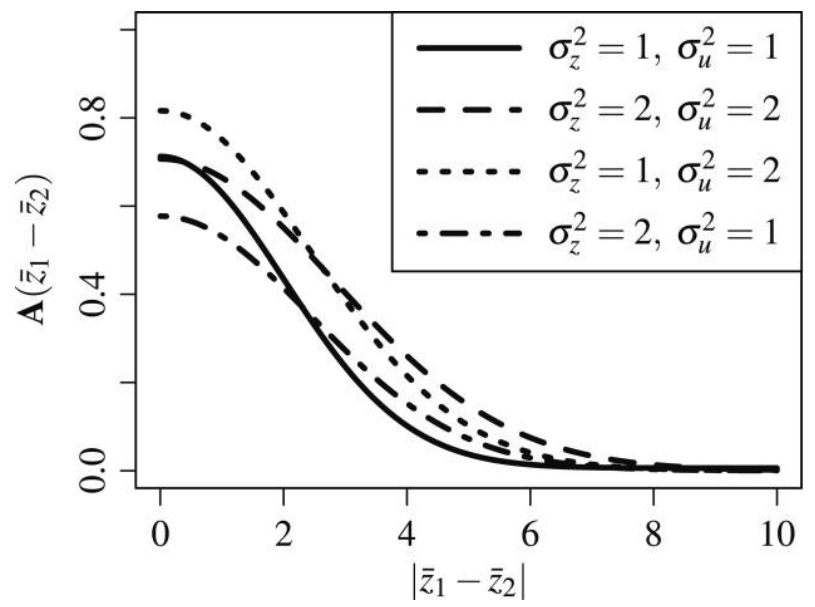

Figure B1: Overall competition coefficient, $\mathbf{A}\left(\bar{z}_{1}-\bar{z}_{2}\right)$ (eq. [B1], with $a_{12}=1$ ), as a function of the difference in mean phenotype between the species. Competition is strongest when the two species have the same mean phenotype. It decreases slowly as the difference in mean phenotype increases, as long as there is still substantial overlap in the species requirements (relatively large $\sigma_{u}^{2}$ and $\sigma_{z}^{2}$ ). When the phenotypes differ more, interspecific competition decreases toward zero.

\section{Literature Cited}

Bassar, R. D., A. Lopez-Sepulcre, M. R. Walsh, M. M. Turcotte, M. Torres-Mejia, and D. N. Reznick. 2010. Bridging the gap between ecology and evolution: integrating density regulation and life-history evolution. Year in Evolutionary Biology 1206:17-34.

Bolnick, D. I., P. Amarasekare, M. S. Araújo, R. Burger, J. M. Levine, M. Novak, V. H. W. Rudolf, S. J. Schreiber, M. C. Urban, and D. A. Vasseur. 2011. Why intraspecific trait variation matters in community ecology. Trends in Ecology \& Evolution 26:183-192.

$\rightarrow$ Brown, W. L., Jr., and E. O. Wilson. 1956. Character displacement. Systematic Zoology 5:49-64.

Butchart, S. H. M., M. Walpole, B. Collen, A. van Strien, J. P. W. Scharlemann, R. E. A. Almond, J. E. M. Baillie, et al. 2010. Global biodiversity: indicators of recent declines. Science 328:1164-1168.

$\rightarrow$ Case, T. J., and M. L. Taper. 2000. Interspecific competition, environmental gradients, gene flow, and the coevolution of species' borders. American Naturalist 155:583-605.

Chase, J. M., and M. A. Leibold. 2003. Ecological niches: linking classical and contemporary approaches. University of Chicago Press, Chicago.

$\rightarrow$ Dayan, T., and D. Simberloff. 2005. Ecological and community-wide character displacement: the next generation. Ecology Letters 8:875894.

$\rightarrow$ Doebeli, M. 1996. An explicit genetic model for ecological character displacement. Ecology 77:510-520.

$\rightarrow$ Ellner, S. P., M. A. Geber, and N. G. Hairston Jr. 2011. Does rapid evolution matter? measuring the rate of contemporary evolution and its impacts on ecological dynamics. Ecology Letters 14:603614.

Faaborg, J., R. T. Holmes, A. D. Anders, K. L. Bildstein, K. M. Dugger, S. A. Gauthreaux, P. Heglund, et al. 2010. Recent advances in understanding migration systems of New World land birds. Ecological Monographs 80:3-48. 
Falconer, D. S., and T. F. C. McKay. 1996. Introduction to quantitative genetics. Pearson, Harlow.

$\rightarrow$ Gabriel, J.-P., F. Saucy, and L.-F. Bersier. 2005. Paradoxes in the logistic equation? Ecological Modelling 185:147-151.

$\rightarrow$ Goldberg, E. E., and R. Lande. 2006. Ecological and reproductive character displacement on an environmental gradient. Evolution 60:1344-1357.

$\rightarrow$ Grant, P. R. 1972. Convergent and divergent character displacement. Biological Journal of the Linnean Society 4:39-68.

Grant, P. R., and B. R. Grant. 1996. Finch communities in a cli matically fluctuating environment. Pages 343-390 in M. L. Cody and J. A. Smallwood, eds. Long term studies of vertebrate communities. Academic Press, San Diego, CA.

$\rightarrow-$ 2002. Unpredictable evolution in a 30-year study of Darwin's finches. Science 296:707-711.

$\rightarrow$ - 2006. Evolution of character displacement in Darwin's finches. Science 313:224-226.

$\rightarrow$ Gross, S. J., and T. D. Price. 2000. Determinants of the northern anc southern range limits of a warbler. Journal of Biogeography 27: 869-878.

$\rightarrow$ Hairston, N. G., S. P. Ellner, M. A. Geber, T. Yoshida, and J. A. Fox. 2005. Rapid evolution and the convergence of ecological and evolutionary time. Ecology Letters 8:1114-1127.

$\rightarrow$ Huston, M. A., and S. Wolverton. 2009. The global distribution or net primary production: resolving the paradox. Ecological Monographs 79:343-377.

$\rightarrow$ Irwin, D. E. 2000. Song variation in an avian ring species. Evolution 54:998-1010.

Katti, M., and T. D. Price. 1996. Effects of climate on Palaearctic warblers over-wintering in India. Journal of the Bombay Natural History Society 93:411-427.

$\rightarrow$. 2003. Latitudinal trends in body size among over-wintering leaf warblers (genus Phylloscopus). Ecography 26:69-79.

$\rightarrow$ Lande, R. 1975 . The maintenance of genetic variability by mutation in a polygenic character with linked loci. Genetical Research 26. 221-235.

$\rightarrow-1982$. A quantitative genetic theory of life history evolution Ecology 63:607-615.

$\rightarrow$ MacArthur, R., and R. Levins. 1967. The limiting similarity, con vergence, and divergence of coexisting species. American Naturalist 101:377-385.

$\rightarrow$ Martin, P. R., and T. E. Martin. 2001. Ecological and fitness conse quences of species coexistence: a removal experiment with wood warblers. Ecology 82:189-206.

$\rightarrow$ Palumbi, S. R. 2001. Evolution—humans as the world's greatest evo lutionary force. Science 293:1786-1790.

$\rightarrow$ Pfennig, K. S., and D. W. Pfennig. 2009. Character displacement ecological and reproductive responses to a common evolutionary problem. Quarterly Review of Biology 84:253-276.

$\rightarrow$ Post, D. M., and E. P. Palkovacs. 2009. Eco-evolutionary feedbacks in community and ecosystem ecology: interactions between the ecological theatre and the evolutionary play. Philosophical Transactions of the Royal Society B: Biological Sciences 364:1629-1640.

$\rightarrow$ Price, T. 1991. Morphology and ecology of breeding warblers along an altitudinal gradient in Kashmir, India. Journal of Animal Ecology 60:643-664.

Price, T., and S. Gross. 2005. Correlated evolution of ecological differences among the Old World leaf warblers in the breeding and non-breeding seasons. Pages 359-372 in R. Greenberg and P. Marra, eds. Birds of two worlds. Smithsonian Institution, Washington, DC.

Price, T. D. 1981. The ecology of the greenish warbler, Phylloscopus trochiloides, in its winter quarters. Ibis 123:131-144.

Price, T. D., and M. Kirkpatrick. 2009. Evolutionarily stable range limits set by interspecific competition. Proceedings of the Royal Society B: Biological Sciences 276:1429-1434.

$\rightarrow$ Price, T. D., A. J. Helbig, and A. D. Richman. 1997. Evolution of breeding distributions in the Old World leaf warblers (genus Phylloscopus). Evolution 51:552-561.

Rasmussen, P., and J. Anderton. 2005. Birds of south Asia: the Ripley guide. Lynx Edicions, Barcelona.

Rodenhouse, N. L., T. S. Sillett, P. J. Doran, and R. T. Holmes. 2003. Multiple density-dependent mechanisms regulate a migratory bird population during the breeding season. Proceedings of the Royal Society B: Biological Sciences 270:2105-2110.

$\rightarrow$ Roughgarden, J. 1972. Evolution of niche width. American Naturalist 106:683-718.

$\rightarrow \longrightarrow$. 1976. Resource partitioning among competing species-a coevolutionary approach. Theoretical Population Biology 9:388424.

- 1979. Theory of population genetics and evolutionary ecology: an introduction. Macmillan, New York.

Schluter, D. 2000. The ecology of adaptive radiation. Oxford University Press, Oxford.

$\rightarrow$ Schluter, D., and P. R. Grant. 1984. Determinants of morphological patterns in communities of Darwin's finches. American Naturalist 123:175-196.

$\rightarrow$ Schluter, D., T. D. Price, and P. R. Grant. 1985. Ecological character displacement in Darwin's finches. Science 227:1056-1059.

$\rightarrow$ Schoener, T. W. 1983. Field experiments on interspecific competition. American Naturalist 122:240-285.

- 2011. The newest synthesis: understanding the interplay of evolutionary and ecological dynamics. Science 331:426-429.

$\rightarrow$ Schreiber, S. J., R. Bürger, and D. I. Bolnick. 2011. The community effects of phenotypic and genetic variation within a predator population. Ecology 92:1582-1593.

$\rightarrow$ Sillett, T. S., N. L. Rodenhouse, and R. T. Holmes. 2004. Experimentally reducing neighbor density affects reproduction and behavior of a migratory songbird. Ecology 85:2467-2477.

$\rightarrow$ Slatkin, M. 1980. Ecological character displacement. Ecology 61:163177.

$\rightarrow$ Taper, M. L., and T. J. Case. 1985. Quantitative genetic models for the coevolution of character displacement. Ecology 66:355-371.

$\rightarrow$ Vasseur, D. A., P. Amarasekare, V. H. W. Rudolf, and J. M. Levine. 2011. Eco-evolutionary dynamics enable coexistence via neighbordependent selection. American Naturalist 178:E96-E109.

Associate Editor: Daniel I. Bolnick Editor: Mark A. McPeek 